\title{
Risk Factors for Mortality in Patients with COVID-19: The Kuwait Experience
}

\author{
Mohammad Al Saleh ${ }^{\text {a }}$ Naser Alotaibi $^{\text {b }}$ Kelly Schrapp ${ }^{c}$ Ahmad Alsaber ${ }^{d}$ \\ Jiazhu Pan ${ }^{d}$ Farah Almutairi $^{a}$ Mohammed Abdullah ${ }^{c}$ Wael Aboelhassan ${ }^{e}$ \\ Noor AINasrallah ${ }^{b}$ Bader Al-Bader $^{\text {a }}$ Haya Malhas ${ }^{f}$ Maryam Ramadhan $^{g}$ \\ Mahdy Hamza $^{\text {h }}$ Hassan Abdelnaby ${ }^{i, j}$ Moudhi Alroomi ${ }^{c}$
}

aDepartment of Medicine, Farwaniya Hospital, Farwaniya, Kuwait; ${ }^{b}$ Department of Medicine, Al Adan Hospital, Hadiya, Kuwait; 'Department of Infectious Diseases, Infectious Diseases Hospital, Shuwaikh Medical Area, Kuwait; ${ }^{\mathrm{d} D e p a r t m e n t}$ of Mathematics and Statistics, University of Strathclyde, Glasgow, UK; 'Division of Gastroenterology,

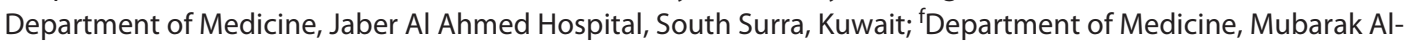
Kabeer Hospital, Jabriya, Kuwait; 9Department of Obstetrics and Gynaecology, Maternity Hospital, Shuwaikh Medical Area, Kuwait; ${ }^{\text {h}}$ Department of Medical Imaging, Al Adan Hospital, Hadiya, Kuwait; 'Department of Endemic and Infectious Diseases, Faculty of Medicine, Suez Canal University, Ismailia, Egypt; jDivision of Gastroenterology, Department of Medicine, Al Sabah Hospital, Shuwaikh Medical Area, Kuwait

\section{Highlights of the Study}

- Risk factors for coronavirus disease-related mortality among patients in Kuwait were evaluated.

- Older age, male sex, and hypertension were significantly linked to mortality.

- Cardiovascular disease and presentation with dyspnea were also risk factors.

\section{Keywords}

COVID-19 · Severe acute respiratory syndrome coronavirus- $2 \cdot$ Mortality $\cdot$ Survival

\begin{abstract}
Objectives: To describe the baseline characteristics and to evaluate the risk factors for in-hospital mortality in patients admitted to hospitals with coronavirus disease (COVID-19) in Kuwait. Subjects and Methods: This retrospective cohort study analyzed data of patients admitted to two hospitals in Kuwait with COVID-19. The outcome was assessed by using multivariable analysis of factors affecting survival and mortality. Results: In 962 patients, the case fatality ratio was
\end{abstract}

9.04\%. The mean age of nonsurvivors was $63.5 \pm 14.8$ years, and most deaths occurred in males (80.5\%). For the whole sample, the source of transmission was significantly related to mortality and the median duration of in-hospital stay was 15 days (interquartile range: $2-52$ days). In patients with high oxygen requirements, the case fatality rate was $96.6 \%$. Multivariable analysis identified age, hypertension, cardiovascular disease (CVD), and dyspnea on presentation as independent risk factors for COVID-19 mortality. Conclusions: The mortality rate was higher in older patients with comorbidities such as hypertension and CVD. Early recognition of highrisk patients may help to improve care and reduce mortality.

(c) 2022 The Author(s).

Published by S. Karger AG, Basel
C 2022 The Author(s).

Published by S. Karger AG, Basel

This is an Open Access article licensed under the Creative Commons Attribution-NonCommercial-4.0 International License (CC BY-NC) (http://www.karger.com/Services/OpenAccessLicense), applicable to the online version of the article only. Usage and distribution for commercial purposes requires written permission.
Correspondence to:

Moudhi Alroomi,moudhisroomi@gmail.com 


\section{Introduction}

Coronavirus disease (COVID-19) is an infection caused by the severe acute respiratory syndrome coronavirus-2 (SARS-CoV-2), which is a novel enveloped, single-stranded, RNA betacoronavirus. The virus is coated by spike $S$, envelope $E$, and membrane $M$ proteins. The $S$ protein on the virus envelope binds to the host cell via the angiotensin-converting enzyme II (ACE II) receptor [1]. ACE II is expressed in multiple tissues of the human body, including the lungs, small intestines, kidneys, heart, thyroid, and adipose tissue. This may explain the multiorgan involvement, and hence presentations observed in COVID-19 range from asymptomatic infections to severe life-threatening disease with multiorgan involvement and death [2].

In December 2019, the first case of COVID-19 was identified in the city of Wuhan, China. The virus then started to spread and infected about 85,000 patients in China. In the early 2020, the virus spread to many countries, and in March 2020, COVID-19 was declared a pandemic by the World Health Organization [3], affecting around 252.2 million people and resulting in 5.1 million deaths worldwide [4]. In Kuwait, as of November 11, 2021, 412,936 cases of COVID-19 had been reported, including 2,462 COVID-19 deaths (State of Kuwait, Ministry of Health Data) [5].

Multiple studies have evaluated the risk factors associated with COVID-19-related mortality [6-8]. A retrospective cohort study that was conducted in New York City in the USA showed an increased risk of mortality from COVID-19 in patients with one or more of the following factors: older age, male sex, tachypnea, hypoxia, impaired renal function, elevated troponin, and elevated D-dimer levels [9]. A similar retrospective cohort study was conducted in Kuwait on 1,096 patients admitted to a single center; the study concluded that a more severe infection was associated with age above 50 years, smoking, presence of asthma, a high quick Sequential Organ Failure Assessment score, and elevated levels of inflammatory markers [10].

The purpose of the present study was to identify the underexplored baseline clinical characteristics of patients on presentation that put them at high risk of COVID19-related death [11-16]. Multiple factors were considered in patients admitted to two hospitals with SARSCoV-2 infections in Kuwait.

\section{Subjects and Methods}

\section{Study Design and Participants}

A total of 962 patients diagnosed with COVID-19, above the age of 18 years, were enrolled in this retrospective cohort study. The study was conducted between February 26, 2020 and September 8, 2020. The data were collected from the electronic medical records of two hospitals in Kuwait: Jaber Al-Ahmed Hospital and Al Adan General Hospital [17-19]. During the data entry stage, an electronic case report form was used. The diagnoses of the patients were based on positive results on reverse transcription-polymerase chain reaction assays of nasopharyngeal swabs. The patients received standard care according to the protocols of the Ministry of Health of Kuwait. The Standing Committee for the Coordination of Health and Medical Research at the Ministry of Health in Kuwait approved the protocol of this study and waived the requirement for informed consent (Institutional Review Board Number 2020/1422).

\section{Definitions}

The intended outcome was COVID-19-related death, based on ICD 10 code U07.1. The following variables were assessed: sociodemographic characteristics, body mass index, smoking status, source of infection, underlying comorbidities, clinical presentations, use of certain medications before admission, oxygen requirement, duration of intensive care unit (ICU), and in-hospital stay. Patients who were on immunosuppressive therapy were included in the immunosuppressed group. Patients with underlying obstructive or restrictive lung disease were grouped under chronic lung disease. The patients' oxygen requirement was classified into three groups: high, low, and none. The high requirement group included patients on extracorporeal membrane oxygenation, invasive ventilation, noninvasive ventilation, or high-flow oxygen; whereas the low requirement group included patients who required oxygen via a nasal canula or a nonrebreather mask.

\section{Statistical Analysis}

The data collected were analyzed using descriptive statistical methods (mean and standard deviation) and the independent samples $t$ tests. The $\chi^{2}$ test or Fisher's exact test was used for comparisons of the categorical data, as appropriate. Cox regression and KaplanMeier method were performed to analyze mortality risk by sex; hazard ratio values of $>1$ represented an increased risk of death. The logrank test was used to compare the survival curves. Logistic regression was used to estimate odd ratios and was employed in the multivariable model. For identifying predictors of mortality, the multivariable model was constructed using the stepwise backward elimination approach. Statistical analyses were performed using SPSS Version 26 (IBM Corp., Armonk, NY, USA) and R statistics version 4.0.3 (R Foundation for Statistical Computing, Vienna, Austria).

\section{Results}

\section{Sociodemographic and Baseline Characteristics}

Data from 962 patients who were admitted to the Jaber Al-Ahmed Hospital and Al Adan General Hospital in Kuwait were included in the analysis. Table 1 shows the sociodemographic characteristics of the study participants. 
Table 1. Baseline characteristics of the study participants

\begin{tabular}{|c|c|c|c|c|c|}
\hline & $\begin{array}{l}\text { All } \\
(N=962)\end{array}$ & $\begin{array}{l}\text { Survivor } \\
(N=875)\end{array}$ & $\begin{array}{l}\text { Nonsurvivor } \\
(N=87)\end{array}$ & $p$ value & $N$ \\
\hline Age, mean $\pm S D$, years & $50.2(15.9)$ & $48.9(15.4)$ & $63.5(14.8)$ & $<0.001$ & 962 \\
\hline $\mathrm{BMI}$, mean $\pm \mathrm{SD}, \mathrm{kg} / \mathrm{m}^{2}$ & $29.0(6.18)$ & $29.1(6.28)$ & $28.2(5.31)$ & 0.221 & 606 \\
\hline \multicolumn{6}{|l|}{ Sex } \\
\hline Female & 344 (35.9) & $327(37.5)$ & $17(19.5)$ & \multirow[t]{2}{*}{0.001} & \multirow[t]{2}{*}{959} \\
\hline Male & $615(64.1)$ & $545(62.5)$ & $70(80.5)$ & & \\
\hline \multicolumn{6}{|l|}{ Smoking } \\
\hline Current smoker & $38(14.1)$ & $32(13.8)$ & $6(15.8)$ & \multirow[t]{3}{*}{0.694} & \multirow[t]{3}{*}{270} \\
\hline Ex-smoker & $28(10.4)$ & $23(9.91)$ & $5(13.2)$ & & \\
\hline Never smoked & $204(75.6)$ & $177(76.3)$ & $27(71.1)$ & & \\
\hline \multicolumn{6}{|l|}{ Source of transmission } \\
\hline Community & $346(40.2)$ & $305(39.4)$ & $41(47.7)$ & \multirow[t]{5}{*}{$<0.001$} & \multirow[t]{5}{*}{860} \\
\hline Contact & $386(44.9)$ & $349(45.1)$ & $37(43.0)$ & & \\
\hline Healthcare worker & $22(2.56)$ & $22(2.84)$ & $0(0.00)$ & & \\
\hline Hospital acquired & $11(1.28)$ & $6(0.78)$ & $5(5.81)$ & & \\
\hline Imported & $95(11.0)$ & $92(11.9)$ & $3(3.49)$ & & \\
\hline Admission to discharge, days [IQR] & $15.0[2.00 ; 52.0]$ & $14.0[2.00 ; 47.0]$ & $23.0[5.00 ; 73.5]$ & $<0.001$ & 950 \\
\hline Hypertension & $324(33.7)$ & $263(30.1)$ & $61(70.1)$ & $<0.001$ & 962 \\
\hline Diabetes mellitus & $335(34.8)$ & $288(32.9)$ & $47(54.0)$ & $<0.001$ & 962 \\
\hline Cardiovascular disease & $79(8.21)$ & $60(6.86)$ & $19(21.8)$ & $<0.001$ & 962 \\
\hline Chronic lung disease & $87(9.04)$ & $82(9.37)$ & $5(5.75)$ & 0.353 & 962 \\
\hline Chronic kidney disease & $43(4.47)$ & $32(3.66)$ & $11(12.6)$ & 0.001 & 962 \\
\hline Immunosuppression & $16(1.66)$ & $11(1.26)$ & $5(5.75)$ & 0.011 & 962 \\
\hline
\end{tabular}

The values are $N(\%)$ unless specified otherwise. BMI, body mass index; IQR, interquartile range; SD, standard deviation.

The case fatality ratio, defined as the incidence proportion of death in patients, was $9.04 \%$. The mean age of the nonsurvivors was 63.5 years and was significantly higher than that of the survivors. Of the fatal cases, $80.5 \%$ of the patients were male and $47.7 \%$ reported community transmission. The proportion of hospital-acquired COVID-19 was higher in this group (5.81\% vs. $0.78 \%)$. The median duration of in-hospital stay for nonsurvivors was 23.0 days (interquartile range: 5.0-73.5 days). Coexisting hypertension, diabetes mellitus (DM), cardiovascular disease (CVD), chronic kidney disease, or immunosuppression were significant risk factors for mortality.

\section{Clinical Presentations}

The association between mortality due to COVID-19 and clinical presentation is shown in Table 2. Among patients who died with COVID-19, 2.3\% were asymptomatic $(p<0.001)$. History of fever was the most commonly reported symptom among nonsurvivors $(71.3 \%)$ and was significantly associated with mortality in patients with COVID-19. Dyspnea at the time of presentation was also associated with increased mortality $(p<0.001)$.

\section{Treatment Characteristics}

Table 3 summarizes the associations between multiple treatment modalities and mortality. Sixteen (25.4\%) patients in the mortality group reported current use of ACE inhibitors compared to $71(9.31 \%)$ in the survival group $(p<0.001)$. Among patients admitted with COVID-19 who were using statins, $182(23.2 \%)$ survived and 37 (52.1\%) died $(p<0.001)$. However, current use of angiotensin II receptor blockers was not associated with mortality $(p=0.293)$. Patients with higher oxygen requirements had a higher mortality $(p<0.001)$ : the mortality rate among patients in the high oxygen requirement group was $96.6 \%$, compared with $2.3 \%$ among those in the low oxygen requirement group, and $1.15 \%$ among those who did not need oxygen. The mortality rate was also significantly higher in patients with longer ICU stays $(p=0.011)$.

\section{Sex and Mortality}

The risk of death was lower in females in the Cox proportional hazards regression (hazard ratio: $0.27, p<$ 0.001 ) and the Kaplan-Meier survival analysis (Fig. 1). 
Table 2. Associations between COVID-19-related mortality and multiple clinical presentations

\begin{tabular}{|c|c|c|c|c|c|}
\hline & $\begin{array}{l}\text { All } \\
(N=962)\end{array}$ & $\begin{array}{l}\text { Survivor } \\
(N=875)\end{array}$ & $\begin{array}{l}\text { Nonsurvivor } \\
(N=87)\end{array}$ & $p$ value & $N$ \\
\hline Asymptomatic & $155(16.1)$ & $153(17.5)$ & $2(2.30)$ & $<0.001$ & 962 \\
\hline Headache & $100(10.4)$ & $92(10.5)$ & $8(9.20)$ & 0.841 & 962 \\
\hline Sore throat & $93(9.67)$ & $86(9.83)$ & $7(8.05)$ & 0.729 & 962 \\
\hline Fever & $547(56.9)$ & $485(55.4)$ & $62(71.3)$ & 0.006 & 962 \\
\hline Dry cough & 459 (47.7) & $416(47.5)$ & $43(49.4)$ & 0.824 & 962 \\
\hline Productive cough & $68(7.07)$ & $64(7.31)$ & $4(4.60)$ & 0.469 & 962 \\
\hline SOB & $309(32.1)$ & $250(28.6)$ & $59(67.8)$ & $<0.001$ & 962 \\
\hline Fatigue or myalgia & $216(22.5)$ & $198(22.6)$ & $18(20.7)$ & 0.781 & 962 \\
\hline Diarrhea & $113(11.7)$ & $104(11.9)$ & $9(10.3)$ & 0.802 & 962 \\
\hline Nausea & $60(6.24)$ & $56(6.40)$ & $4(4.60)$ & 0.667 & 962 \\
\hline Vomiting & $59(6.13)$ & $57(6.51)$ & $2(2.30)$ & 0.184 & 962 \\
\hline Change of taste or smell & $34(3.53)$ & $30(3.43)$ & $4(4.60)$ & 0.540 & 962 \\
\hline
\end{tabular}

The values are $N(\%)$ unless specified otherwise. COVID-19, coronavirus disease; SOB, shortness of breath.

Table 3. Association of medications and COVID-19 treatment modalities with mortality due to COVID-19

\begin{tabular}{llllrr}
\hline & $\begin{array}{l}\text { All } \\
(N=887)\end{array}$ & $\begin{array}{l}\text { Survivor } \\
(N=800)\end{array}$ & $\begin{array}{l}\text { Nonsurvivor } \\
(N=87)\end{array}$ & $p$ value & $N$ \\
\hline Receiving ACE inhibitors & $87(10.5)$ & $71(9.31)$ & $16(25.4)$ & $<0.001$ & 826 \\
Receiving ARBs & $110(13.3)$ & $99(12.9)$ & $11(18.6)$ & 0.293 & 826 \\
Receiving statin & $219(25.6)$ & $182(23.2)$ & $37(52.1)$ & $<0.001$ & 855 \\
$\begin{array}{l}\text { Oxygen requirements } \\
\quad \text { High oxygen requirement }\end{array}$ & $139(15.7)$ & $55(6.88)$ & $84(96.6)$ & $<0.001$ & 887 \\
$\quad$ Low oxygen requirement & $249(28.1)$ & $247(30.9)$ & $2(2.3)$ & & \\
$\quad$ None & $499(56.3)$ & $498(62.3)$ & $1(1.15)$ & & \\
ICU length of stay, mean \pm SD, days & $18.0(17.4)$ & $14.1(16.1)$ & $21.2(17.9)$ & 0.011 & 151 \\
\hline
\end{tabular}

The values are $N(\%)$ unless specified otherwise. ACE, angiotensin-converting enzyme; ARBs, angiotensin II receptor blockers; COVID-19, coronavirus disease; ICU, intensive care unit; SD, standard deviation.

\section{Multivariable Logistic Regression Model}

Risk factors for death were assessed using a multivariable logistic regression model that included age, hypertension, DM, CVD, dyspnea at time of presentation, current ACE inhibitor use, and current statin use (Table 4). The data showed that age (adjusted odds ratio [aOR]: $1.05,95 \%$ confidence interval [CI]: $1.03-1.08, p<0.001$ ), hypertension (aOR: $2.25,95 \%$ CI: $1.10-4.67, p=0.028$ ), CVD (aOR: 2.45 , 95\% CI: 1.10-5.43, $p=0.027$ ), and dyspnea (aOR: $4.07,95 \%$ CI: $2.27-7.52, p<0.001$ ) were significantly associated with mortality. However, DM ( $p=$ $0.697)$, ACE inhibitor use $(p=0.121)$, and statin use $(p=$ 0.142 ) had no significant effect on mortality.

\section{Discussion}

In this study, multiple risk factors for mortality were considered in 962 patients with COVID-19. The data showed that patients who died due to COVID-19 were likely to be older. This finding is consistent with the results of previous studies that showed a higher mortality rate in older patients with COVID-19, particularly in those aged $\geq 60$ years $[20,21]$. There was also a significant relationship between sex and mortality due to COVID-19. A recent study showed that males had a higher risk of severe acute respiratory distress syndrome than females, with the mortality rates ranging from $59 \%$ to $75 \%$. This may be attributed to the higher expression of ACE II in males, which is the main receptor for the binding of SARS-CoV-2 to host cells [22]. 
Table 4. Multivariable logistic regression model between certain variables and in-hospital mortality due to COVID-19

\begin{tabular}{llllll}
\hline & & Survivor & Nonsurvivor & Crude OR $(95 \% \mathrm{Cl}, p)$ & Adjusted OR (95\% Cl, $p$ ) \\
\hline Age & Mean (SD) & $48.9(15.4)$ & $63.5(14.8)$ & $1.06(1.05-1.08, p<0.001)$ & $1.05(1.03-1.08, p<0.001)$ \\
Hypertension & Yes & $263(81.2)$ & $61(18.8)$ & $5.46(3.41-8.97, p<0.001)$ & $2.25(1.10-4.67, p=0.028)$ \\
DM & Yes & $288(86.0)$ & $47(14.0)$ & $2.39(1.54-3.75, p<0.001)$ & $1.14(0.59-2.23, p=0.697)$ \\
CVD & Yes & $60(75.9)$ & $19(24.1)$ & $3.80(2.10-6.63, p<0.001)$ & $2.45(1.10-5.43, p=0.027)$ \\
SOB & Yes & $250(80.9)$ & $59(19.1)$ & $5.27(3.31-8.56, p<0.001)$ & $4.07(2.27-7.52, p<0.001)$ \\
ACE inhibitors & Yes & $71(81.6)$ & $16(18.4)$ & $3.32(1.74-6.05, p<0.001)$ & $1.84(0.84-3.93, p=0.121)$ \\
Statin & Yes & $182(83.1)$ & $37(16.9)$ & $3.60(2.20-5.92, p<0.001)$ & $0.56(0.25-1.20, p=0.142)$ \\
\hline
\end{tabular}

The values are $N(\%)$ unless specified otherwise. $\mathrm{ACE}$, angiotensin-converting enzyme; $\mathrm{Cl}$, confidence interval; COVID-19, coronavirus disease; CVD, cardiovascular disease; DM, diabetes mellitus; OR, odds ratio; SD, standard deviation; SOB, shortness of breath.

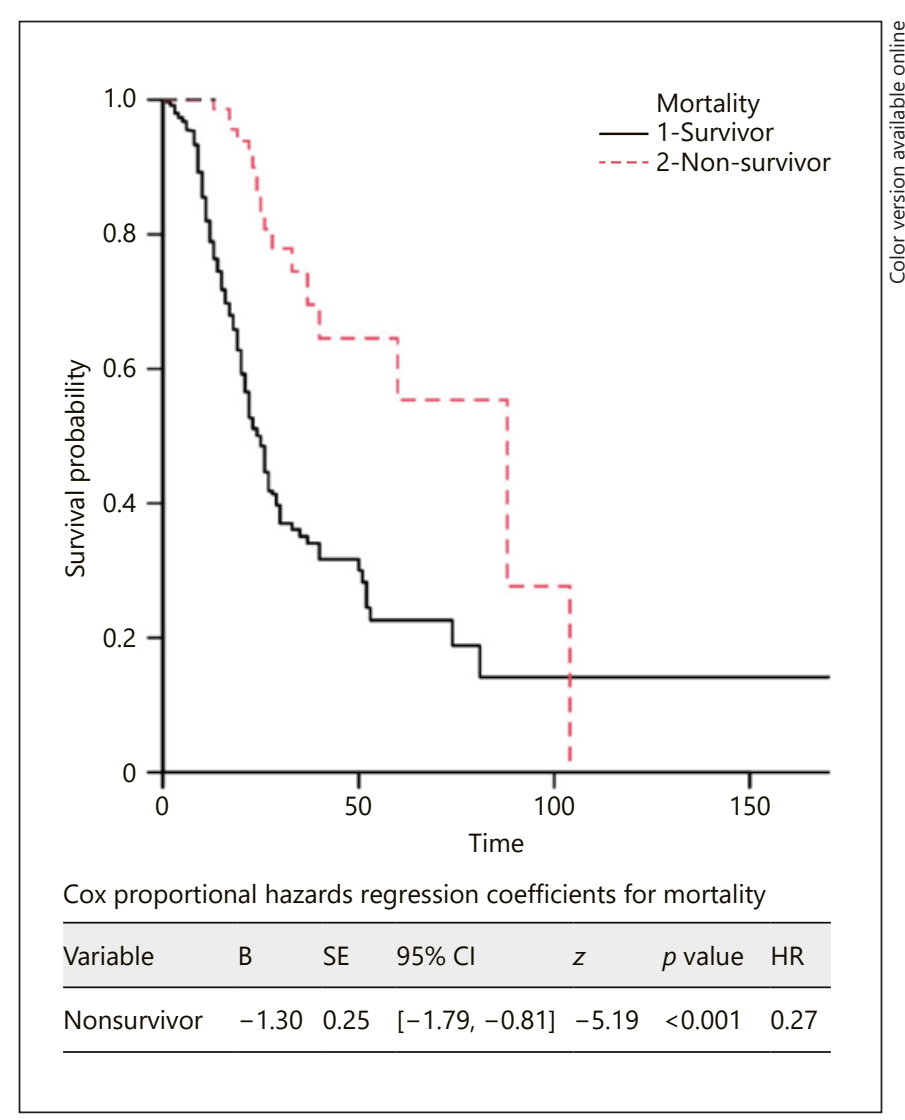

Fig. 1. Kaplan-Meier survival probability plot for mortality. Female sex was used to represent a hazard event and male sex was used to indicate survival. $x$-axis: days since admission.

We also investigated the relationship between existing comorbidities and mortality due to COVID-19. A higher mortality rate was observed in patients with hypertension; this finding is similar to the results of a recent pro- spective cohort study that was conducted in Spain, in which hypertension was the most reported previous comorbidity in the nonsurvivors' group $(61.10 \%, p<0.0001)$ [23]. Furthermore, in our study, a significant relationship was also observed between DM and mortality. A study conducted in Mexico showed a significant relationship between mortality and diabetes in patients hospitalized with COVID-19 (aOR: 1.28, 95\% CI: 1.23-1.34, $p<$ 0.0001) [24]. Additionally, comorbid CVD, chronic kidney disease, and immunosuppression were also associated with an increased mortality in COVID-19; however, chronic lung disease was not significantly associated with an increased risk of death. This finding was contrary to a previous study that showed patients who were hospitalized with COVID-19 and had comorbid chronic obstructive pulmonary disease had a higher mortality (aOR: 1.26, 95\% CI: $1.15-1.38, p<0.0001)$ [24].

In the present study, in-hospital oxygen therapy was also associated with higher mortality. This result was in accordance with a recent study conducted in China showing that nonsurvivors were more likely to have received oxygen therapy (high-flow nasal cannula [89\%, $p<0.001]$, noninvasive mechanical ventilation [57\%, $p<0.001]$, invasive mechanical ventilation $[35 \%, p<$ $0.001]$, extracorporeal membrane oxygenation $[1 \%, p=$ $0.005]$ ] [25]. Mortality was also significantly associated with the duration of ICU stay. This finding was also observed in a cohort study conducted in Brazil, which showed a mortality rate of $18.9 \%$ in patients admitted to the ICU $(p<0.001)$, with a median interval between ICU admission and death/end of the survey of 12.5 days $(p<0.001)$ [26]. An explanation for this finding may be the late presentation of patients with complicated COVID-19 to the hospital, which necessitated earlier ICU admission. 
This study has several limitations. The data were obtained from electronic medical records, which are prone to underdocumentation of history and presentation. This was especially evident for smoking; data on smoking history were missing for more than $50 \%$ of patients. Another limitation is that despite a significant association between DM and COVID-19-related mortality in the univariable analysis, the sample size was too small to confirm an effect in the multivariable analysis. Our study was performed retrospectively because a prospective study design would require a strict timeframe and follow-up, which was not possible for several reasons, including the burden of the outbreak on the health system.

\section{Conclusions}

This study highlighted certain baseline characteristics as predictors of COVID-19 mortality in Kuwait. Older age, male sex, hypertension, CVD, and presentation with dyspnea were significantly associated with increased risk of death in hospitalized COVID-19 patients. Healthcare authorities should identify these factors at the time of diagnosis to improve patient care and outcomes.

\section{Acknowledgment}

The authors thank Dr Danah Alothman for her support in manuscript review.

\section{Statement of Ethics}

The study protocol was reviewed and approved by the Standing Committee for the Coordination of Health and Medical Research of the Ministry of Health, Kuwait (Institutional Review Board Number: 2020/1422). The requirement of written informed consent was covered by the same committee.

\section{Conflict of Interest Statement}

The authors have no conflicts of interest to declare.

\section{Funding Sources}

This research did not receive any specific grant from funding agencies in the public, commercial, or not-for-profit sectors.

\section{Author Contributions}

M.A. designed the study. M.A.S., N.A., K.S., F.A., and M.A. wrote the manuscript. A.A. and J.P. analyzed the data. The remaining authors collected the data and took responsibility for the integrity and accuracy of the data. All authors have read and approved the manuscript.

\section{Data Availability Statement}

The data that support the results of the study are available upon request from the corresponding author. The data are not publicly available due to ethical restrictions.

\section{References}

1 Zheng YY, Ma YT, Zhang JY, Xie X. COVID-19 and the cardiovascular system. Nat Rev Cardiol. 2020 May;17(5):259-60.

2 Cevik M, Kuppalli K, Kindrachuk J, Peiris M. Virology, transmission, and pathogenesis of SARS-CoV-2. BMJ. 2020 Oct 23;371:m3862.

3 World Health Organization. Coronavirus disease (COVID-19) Situation Report: 52 [cited 2020 Dec 30]. Available from: https:// www.who.int/docs/default-source/coronaviruse/situation-reports/20200312-sitrep52-covid-19.pdf?sfvrsn=e2bfc9c0_4.

4 Johns Hopkins University and Medicine. COVID-19 dashboard by the center for systems science and engineering (CSSE) at Johns Hopkins University (JHU) [cited $2021 \mathrm{Nov}$ 11]. Available from: https://coronavirus.jhu. edu/map.html.

5 State of Kuwait, Ministry of Health. COVID-19 updates [cited 2021 Nov 11]. Available from: https://corona.e.gov.kw.
6 Albitar O, Ballouze R, Ooi JP, Sheikh Ghadzi SM. Risk factors for mortality among COVID-19 patients. Diabetes Res Clin Pract. 2020 Aug 1;166:108293.

7 Tian W, Jiang W, Yao J, Nicholson CJ, Li RH, Sigurslid HH, et al. Predictors of mortality in hospitalised COVID-19 patients: a systematic review and meta-analysis. J Med Virol. 2020 Oct;92(10):1875-83.

8 Escobedo-de la Peña J, Rascón-Pacheco RA, de Jesús Ascencio-Montiel I, GonzálezFigueroa E, Fernández-Gárate JE, MedinaGómez OS, et al. Hypertension, diabetes and obesity, major risk factors for death in patients with COVID-19 in Mexico. Arch Med Res. 2021 May 1;52(4):443-9.

9 Mikami T, Miyashita H, Yamada T, Harrington $\mathrm{M}$, Steinberg D, Dunn A, et al. Risk factors for mortality in patients with $\mathrm{CO}$ VID-19 in New York City. J Gen Intern Med. 2021;36:17-26.
10 Almazeedi S, Al-Youha S, Jamal MH, AlHaddad M, Al-Muhaini A, Al-Ghimlas F, et al. Characteristics, risk factors and outcomes among the first consecutive 1096 patients diagnosed with COVID-19 in Kuwait. EClinicalMedicine. 2020;24:100448.

11 Alshukry A, Ali H, Ali Y, Al-Taweel T, AbuFarha M, AbuBaker J, et al. Clinical characteristics of coronavirus disease 2019 (COVID-19) patients in Kuwait. PLoS One. 2020 Nov 20;15(11):e0242768.

12 Jamal MH, Doi SA, AlYouha S, Almazeedi S, Al-Haddad M, Al-Muhaini A, et al. A biomarker based severity progression indicator for $\mathrm{CO}$ VID-19: the Kuwait prognosis indicator score. Biomarkers. 2020 Nov 16;25(8):641-8.

13 Ayed M, Borahmah AA, Yazdani A, Sultan A Mossad A, Rawdhan H. Assessment of clinical characteristics and mortality-associated factors in COVID-19 critical cases in Kuwait. Med Princ Pract. 2021;30(2):185-92. 
14 Alshukry A, Abbas MB, Ali Y, Alahmad B, AlShammari AA, Alhamar G, et al. Clinical characteristics and outcomes of COVID-19 patients with diabetes mellitus in Kuwait. Heliyon. 2021 Apr 1;7(4):e06706.

15 Al-Sabah S, Al-Haddad M, Al-Youha S, Jamal M, Almazeedi S. COVID-19: impact of obesity and diabetes on disease severity. Clin Obes. 2020 Dec;10(6):e12414.

16 ElAbd R, AlTarrah D, AlYouha S, Bastaki H, Almazeedi S, Al-Haddad M, et al. Angiotensin-converting enzyme (ACE) inhibitors and angiotensin receptor blockers (ARB) are protective against ICU admission and mortality for patients with COVID-19 disease. Front Med. 2021 Mar 4;8:600385.

17 Al-Jarallah M, Rajan R, Saber AA, Pan J, AlSultan AT, Abdelnaby H, et al. In-hospital mortality in SARS-CoV-2 stratified by hemoglobin levels: a retrospective study. EJHaem. 2021. Online ahead of print.
18 Al-Jarallah M, Rajan R, Saber AA, Pan J, AlSultan AT, Abdelnaby H, et al. In-hospital Mortality in SARS-CoV-2 stratified by serum 25-hydroxy-vitamin D levels: a retrospective study. J Med Virol. 2021 Oct;93(10):5880-5.

19 Alroomi M, Rajan R, Omar AA, Alsaber A, Pan J, Fatemi M, et al. Ferritin level: a predictor of severity and mortality in hospitalized COVID-19 patients. Immun Inflamm Dis. 2021 Dec;9(4):1648-55.

20 Zhou F, Yu T, Du R, Fan G, Liu Y, Liu Z, et al. Clinical course and risk factors for mortality of adult inpatients with COVID-19 in Wuhan, China: a retrospective cohort study. Lancet. 2020 Mar 28;395(10229):1054-62.

21 CDC COVID-19 Response Team. Severe outcomes among patients with coronavirus disease 2019 (COVID-19) - United States, February 12-March 16, 2020. MMWR Morb Mortal Wkly Rep. 2020 Mar 27;69(12):343-6.
22 Bienvenu LA, Noonan J, Wang X, Peter K. Higher mortality of COVID-19 in males: sex differences in immune response and cardiovascular comorbidities. Cardiovasc Res. 2020 Dec 1;116(14):2197-206.

23 Laguna-Goya R, Utrero-Rico A, Talayero P, Lasa-Lazaro M, Ramirez-Fernandez A, Naranjo L, et al. IL-6-based mortality risk model for hospitalized patients with COVID-19. J Allergy Clin Immunol. 2020 Oct;146(4):799-807.

24 Parra-Bracamonte GM, Lopez-Villalobos N, Parra-Bracamonte FE. Clinical characteristics and risk factors for mortality of patients with COVID-19 in a large data set from Mexico. Ann Epidemiol. 2020 Dec;52:93-8.e2.

25 Chen L, Yu J, He W, Chen L, Yuan G, Dong F, et al. Risk factors for death in 1859 subjects with COVID-19. Leukemia. 2020 Aug;34(8):2173-83.

26 Sousa GJB, Garces TS, Cestari VRF, Florêncio RS, Moreira TMM, Pereira MLD. Mortality and survival of COVID-19. Epidemiol Infect. 2020 Jun 25;148:e123. 